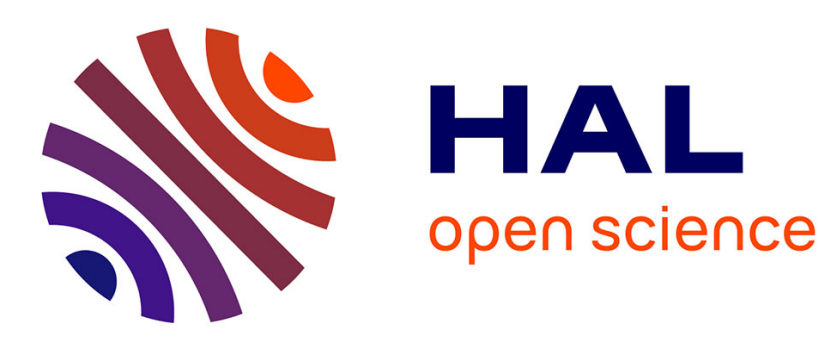

\title{
Effect of variable mass density on the kinematics of scalar gradient
}

\author{
M Gonzalez, P Paranthoën
}

\section{To cite this version:}

M Gonzalez, P Paranthoën. Effect of variable mass density on the kinematics of scalar gradient. Physics of Fluids, 2011, 23, pp.075107. 10.1063/1.3609281 . hal-01412404

\author{
HAL Id: hal-01412404 \\ https://hal.science/hal-01412404
}

Submitted on 8 Dec 2016

HAL is a multi-disciplinary open access archive for the deposit and dissemination of scientific research documents, whether they are published or not. The documents may come from teaching and research institutions in France or abroad, or from public or private research centers.
L'archive ouverte pluridisciplinaire HAL, est destinée au dépôt et à la diffusion de documents scientifiques de niveau recherche, publiés ou non, émanant des établissements d'enseignement et de recherche français ou étrangers, des laboratoires publics ou privés. 


\title{
Effect of variable mass density on the kinematics of scalar gradient
}

\author{
M. Gonzalez ${ }^{\mathrm{a})}$ and P. Paranthoën \\ CNRS, UMR 6614/CORIA, Site universitaire du Madrillet, 76801 Saint-Etienne du Rouvray, France
}

(Received 3 March 2011; accepted 7 June 2011; published online 21 July 2011)

\begin{abstract}
Analysis of the equations for the orientation and norm of the gradient of a passive scalar shows that the kinematics of the scalar gradient may be deeply altered by non-solenoidal effects felt through the velocity gradient. While these effects are explicitly expressed in the equation for the gradient norm, the orientation equations are unaltered as compared to the incompressible case. In two-dimensional flows the behavior of the scalar gradient is governed by both the strain persistence parameter, $r$, and the ratio, $\delta / \sigma$, of velocity divergence to the norm of the deviatoric part of the strain tensor. In particular, in the dilatational case, while large dilatation $(\delta / \sigma>1)$ unconditionally lessens the scalar gradient, moderate dilatation $(0<\delta / \sigma<1)$ needs effective rotation $(r \neq 0)$ to drive the scalar gradient to decrease. In three-dimensional flows the analytic study needs the assumption that vorticity is aligned with a strain principal axis and leads to a more complex picture based on the strain persistence parameter, the velocity divergence and the strain eigenvalue corresponding to the strain direction parallel to vorticity. In this case, too, for moderate non-solenoidal dilatational effects, rotation appears to oppose the rise of the scalar gradient norm. Possible inferences of the analysis relevant to reacting flows with heat release are briefly discussed (c) 2011 American Institute of Physics. [doi:10.1063/1.3609281]
\end{abstract}

\section{INTRODUCTION}

Understanding and possibly controlling mixing phenomena in fluids needs a precise knowledge of velocity field properties and flow structure. Complete homogenization is achieved by molecular diffusion, but the whole process may be hastened by a flow field producing small scales of the scalar to be mixed, a mechanism that can be viewed as the expression of the mechanical action of the velocity gradient upon the scalar gradient. A number of works addressed the effect of velocity gradient on passive scalar gradient properties in constant-density flows. ${ }^{1-4}$ Mixing processes in fluids, however, frequently take place together with spatial variations of mass density which alter velocity gradient properties. This occurs, for instance, in stratified environmental and engineering flows or in reacting flows where local heat release results in steep density gradients. A relevant issue, then, is to explain how mass density gradients may play on stirring properties of the flow through the local features of the velocity gradient.

The influence of variable density upon velocity gradient properties has been analyzed in some studies. The case of compressible flows is considered in the classification of local flow topologies devised by Chong et al. ${ }^{5}$ Erlebacher and Sar$\operatorname{kar}^{6}$ and Lee et $\mathrm{al}^{7}$ investigated the structure of the velocity gradient tensor in compressible turbulence. Porter et al. ${ }^{8}$ and Miura $^{9}$ analyzed the vorticity structures, while Pirozzoli and Grasso $^{10}$ and Suman and Girimaji ${ }^{11}$ examined the effect of compressibility on the local flow topology through the velocity gradient invariants. Hua et al. ${ }^{12}$ found that stratification

\footnotetext{
a) Author to whom correspondence should be addressed. Electronic mail: Michel.Gonzalez@coria.fr.
}

influences the topology of stirring of geostrophic turbulence through the velocity gradient and Diamessis and Nomura ${ }^{13}$ showed that it results in a tight interaction of strain, vorticity and density gradient in homogeneous sheared turbulence.

The effect of mass density variations was specially emphasized in combustion flows in which chemical reactions involve a strong local heat release. Scalar dissipation, in particular, was found to be altered by heat release ${ }^{14-16}$ thus suggesting that the velocity gradient/scalar gradient interaction is affected by density variations. More specifically, Swaminathan and Grout ${ }^{15}$ - see also subsequent studies ${ }^{17,18}$-and Mura et al. ${ }^{16}$ investigated the effect of heat release upon the stretching of the gradient of a reacting scalar in premixed flames. In nonpremixed flames, Nomura and Elghobashi ${ }^{19}$ and Boratav et al. ${ }^{20,21}$ investigated the properties of the velocity gradient tensor. They studied how strain properties and vorticity alignments are changed by variable density through dilatational and baroclinic effects. In particular, Boratav et $a .^{21}$ showed that the dissipation rate of fuel concentration is indirectly affected by buoyancy through vorticity alignment and also analyzed the change in the properties of the pressure Hessian - which is directly involved in the rotation of strain principal axes $^{22-24}$-resulting from variable density.

Studies in combustion flows have essentially addressed the problem for the gradient of a reacting scalar thus involving complex effects resulting mainly from chemical reactions and from the dependence of mass density variationsthrough heat release - on the chemical consumption of the scalar. By considering the gradient of an inert, passive scalar we get rid of these effects and focus the analysis on those non-solenoidal mechanisms which specifically affect the scalar gradient through the velocity gradient properties. 
Incidentally, the kinematics of the gradient of a passive scalar in a non-solenoidal flow is relevant to the behavior of the mixture fraction gradient in nonpremixed or in stratified combustion. Lapeyre ${ }^{25}$ mentioned the qualitative effect of variable mass density on the gradient of a passive scalar, but it appears that no detailed, analytic study on the subject has been undertaken.

We follow this line in Sec. II which is devoted to the study of the non-solenoidal kinematics of the scalar gradient based on the analysis of the equations for the orientation and norm in both two- and three-dimensional flows. In Sec. III we propose a tentative discussion of the effect of variable mass density upon the scalar gradient in flame fronts derived from the analysis of the two-dimensional case. Conclusion is drawn in Sec. IV.

\section{KINEMATICS OF SCALAR GRADIENT IN A NON-SOLENOIDAL VELOCITY FIELD}

\section{A. Two-dimensional case}

\section{Properties of the strain tensor}

The strain tensor, $S$, is the symmetric part of the velocity tensor and its components are defined as

$$
S_{i j}=\frac{1}{2}\left(\frac{\partial u_{i}}{\partial x_{j}}+\frac{\partial u_{j}}{\partial x_{i}}\right),
$$

where $u_{i}$ are the velocity components. Tensor $S$ can be expressed in terms of its isotropic and deviatoric parts as

$$
S_{i j}=\frac{1}{2} \delta_{i j} S_{\alpha \alpha}+\sigma_{i j}
$$

where $\delta_{i j}$ is the Kronecker symbol and $\sigma_{i j}$ are the components of the deviatoric part of $\boldsymbol{S}$. Tensor $\boldsymbol{\sigma}$ is symmetric and traceless and its components are given by

$$
\begin{gathered}
\sigma_{11}=-\sigma_{22}=\frac{1}{2}\left(\frac{\partial u_{1}}{\partial x_{1}}-\frac{\partial u_{2}}{\partial x_{2}}\right), \\
\sigma_{12}=\sigma_{21}=\frac{1}{2}\left(\frac{\partial u_{1}}{\partial x_{2}}+\frac{\partial u_{2}}{\partial x_{1}}\right) .
\end{gathered}
$$

The trace of tensor $S$ is $S_{\alpha \alpha}=\partial u_{\alpha} / \partial x_{\alpha}=-(1 / \rho) D \rho / D t$ (where $\rho$ is the mass density and $D / D t$ the Lagrangian derivative) and is denoted by $S_{\alpha \alpha}=\delta$.

It is straightforward to show that the characteristic equation for the eigenvalues, $\lambda$, of $S$ is

$$
\lambda^{2}-\delta \lambda+\frac{1}{4}\left(\delta^{2}-\sigma^{2}\right)=0,
$$

where $\sigma=2\left(\sigma_{11}^{2}+\sigma_{12}^{2}\right)^{1 / 2}$. Tensor $S$ has thus one negative and one positive eigenvalues-corresponding, respectively, to compression and extension-if $\delta^{2} / \sigma^{2}<1$; if $\delta^{2} / \sigma^{2}>1$, then the eigenvalues have the same sign, either positive or negative for $\delta>0$ or $\delta<0$, respectively. The eigenvalues are given by $\lambda= \pm \sigma / 2+\delta / 2$ and the incompressible case is of course retrieved for $\delta=0$ which gives $\lambda= \pm \sigma / 2$.

\section{Equations for the scalar gradient}

The equation for the gradient, $\boldsymbol{G}$, of a passive scalar, $\Theta$, is derived from the equation for $\Theta$ which, using the mass conservation equation, is written as

$$
\frac{\partial \Theta}{\partial t}+u_{\alpha} \frac{\partial \Theta}{\partial x_{\alpha}}=\frac{1}{\rho} \frac{\partial}{\partial x_{\alpha}} \rho D \frac{\partial \Theta}{\partial x_{\alpha}},
$$

where $D$, the molecular diffusivity of $\Theta$, is assumed to be constant. Differentiating each side of Eq. (1) with respect to $x_{i}$ gives the equation for the components, $G_{i}$, of the scalar gradient

$$
\begin{aligned}
\frac{\partial G_{i}}{\partial t}+u_{\alpha} \frac{\partial G_{i}}{\partial x_{\alpha}}= & -\frac{\partial u_{\alpha}}{\partial x_{i}} G_{\alpha}+\frac{1}{\rho} \frac{\partial}{\partial x_{\alpha}} \rho D \frac{\partial G_{i}}{\partial x_{\alpha}} \\
& +\frac{D}{\rho} G_{\alpha}\left(\frac{\partial^{2} \rho}{\partial x_{\alpha} \partial x_{i}}-\frac{1}{\rho} \frac{\partial \rho}{\partial x_{i}} \frac{\partial \rho}{\partial x_{\alpha}}\right) .
\end{aligned}
$$

In both equations for the scalar and its gradient possible effects of the gradients of mass density explicitly find expression through the diffusion terms. Restricting the analysis to non-diffusive scalars as already done in previous studies $^{24,26}$ leads to a simpler equation for the components of the scalar gradient,

$$
\frac{\partial G_{i}}{\partial t}+u_{\alpha} \frac{\partial G_{i}}{\partial x_{\alpha}}=-\frac{\partial u_{\alpha}}{\partial x_{i}} G_{\alpha}
$$

In Eq. (3) the possible influence of both spatial and temporal variations of mass density is indirectly felt through the velocity field. It is the purpose of the study to examine how mass density variations play on the kinematics of the scalar gradient through the velocity field and more precisely through the velocity gradient. We assume there is no imposed, mean scalar gradient (Appendix A).

As in the incompressible case, ${ }^{24}$ starting from Eq. (3) with the scalar gradient expressed in polar coordinates as $\boldsymbol{G}=|\boldsymbol{G}|(\cos \theta, \sin \theta)$ leads to the equations for the orientation and norm of the gradient,

$$
\frac{D \zeta}{D \tau}=r-\cos \zeta
$$

$$
\frac{1}{|\mathbf{G}|^{2}} \frac{D|\mathbf{G}|^{2}}{D \tau}=-\left(\sin \zeta+\frac{\delta}{\sigma}\right)
$$

in which $D \tau=\sigma D t$. Variable $\zeta$ defining the scalar gradient orientation is $\zeta=2(\theta+\Phi)$ where $\Phi$ gives the orientation of strain principal axes through $\tan (2 \Phi)=\sigma_{11} / \sigma_{12}$. Parameter $r$ defines strain persistence as the ratio of effective rotation to strain as $r=(\omega+2 D \Phi / D t) / \sigma$ where $\omega$ is the vorticity. ${ }^{24}$ Note that the right-hand side of Eq. (5) — multiplied by $\sigma$-is the exact expression in polar coordinates of the production rate of the gradient norm, $-G_{\alpha} S_{\alpha \beta} G_{\beta} /|\boldsymbol{G}|^{2}$; examining the sign of the growth rate of the gradient norm thus comes to study the sign of the so-called 'stretching term', $-G_{\alpha} S_{\alpha \beta} G_{\beta}$, which plays an essential role in the equation for the dissipation rate of scalar fluctuations. ${ }^{15,16,27}$ 
As mentioned by Lapeyre, ${ }^{25}$ there is no direct influence of mass density variation on the equation for the gradient orientation [Eq. (4)], while the evolution of the gradient norm [Eq. (5)] is directly affected by the ratio $\delta / \sigma$ which measures the non-solenoidal effect with respect to the mechanical action of strain. In fact, the isotropic form of the non-solenoidal part of the strain tensor implies that non-solenoidal effects cannot directly alter the orientation properties of the gradient. Note that since the dynamical variables entering into the definition of the strain persistence parameter, $r$, may be affected by non-solenoidal effects as well, the latter indirectly play on gradient orientation and, then, on gradient norm.

\section{Kinematics of the scalar gradient}

Equation (4) for gradient orientation has the same form as in the solenoidal case and the analysis of the dynamics of gradient orientation in terms of strain persistence ${ }^{24}$ thus remains unchanged. In particular, in the special case where $r=0$ the scalar gradient tends to align with the strain eigenvector corresponding to the smallest eigenvalue; the big difference, this time, is that the latter direction may be an extensive one-instead of being the compressive one in the solenoidal case-which occurs when the strain tensor has two positive eigenvalues, that is, when $\delta^{2} / \sigma^{2}>1$ and $\delta>0$ (Sec. II A). It is also straightforward to show that in the adiabatic regime defined by slow variations of $r$ along the Lagrangian trajectories the analytic solution to Eq. (5) for the gradient norm is derived by multiplying by $\exp \left[-\int_{0}^{\tau}(\delta / \sigma)\left(\tau^{\prime}\right) d \tau^{\prime}\right]$ the solution derived by Lapeyre et $a l .{ }^{24}$ for $\delta=0$. In the simple case where $\delta / \sigma$ is a constant the non-solenoidal effect comes to decrease (if $\delta / \sigma>1$ ) or increase (if $\delta / \sigma<-1$ ) the gradient norm as $\exp [-(\delta / \sigma) \tau]$.

The intermediate case in which $\delta^{2} / \sigma^{2}<1$ is more interesting; the strain tensor has two opposed eigenvalues corresponding respectively to a compressive and an extensive directions, but the kinematics of the scalar gradient significantly departs from what it is in the solenoidal case. This essentially results from the spreading, in the domain of the orientation variable, of the influence of either compressive effects (if $\delta<0$ ) or extensive ones (if $\delta>0$ ). We restrict the discussion to the simple case where $\delta / \sigma$ is a constant-such that $\delta^{2} / \sigma^{2}<1-$. We also assume $\delta>0$ which is relevant, for instance, to reacting flows with heat release.

When strain prevails over effective rotation $\left(r^{2}<1\right)$, in the adiabatic regime analyzed by Lapeyre $e t a l .{ }^{24}$ the scalar gradient orientation has a stable fixed point which is given by $\zeta_{e q}=-\arccos (r)$. When equilibrium is reached in terms of orientation $\zeta \simeq \zeta_{e q}$ and Eq. (5) for the norm of the scalar gradient can thus be rewritten as

$$
\frac{1}{|\boldsymbol{G}|^{2}} \frac{D|\boldsymbol{G}|^{2}}{D \tau}=\left(1-r^{2}\right)^{1 / 2}-\frac{\delta}{\sigma},
$$

which shows that the growth rate of the gradient norm is positive provided that

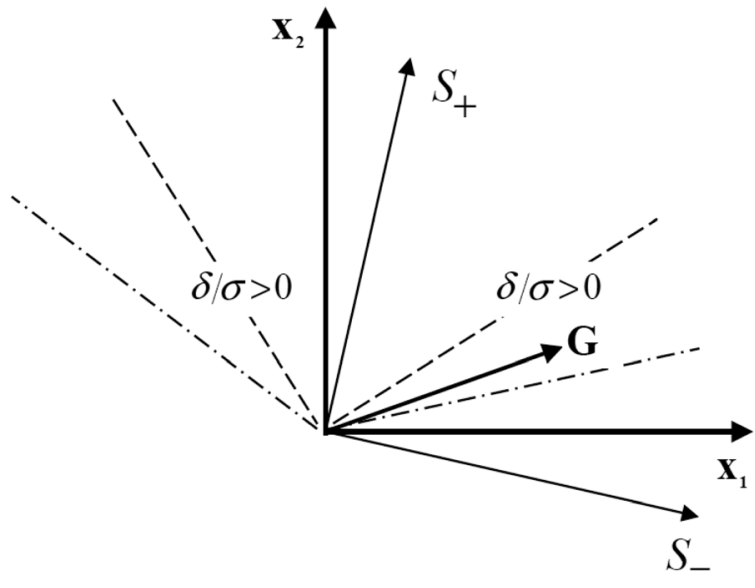

FIG. 1. Spreading of extensional effects in the orientation domain when $\delta / \sigma>0$ (with $\delta^{2} / \sigma^{2}<1$ ); dashed lines are the limits of the extensive-straininfluenced domain in the solenoidal case $(\delta=0)$; dash-dotted lines show the spreading of extensional effects for $\delta / \sigma>0 ; S_{-}$and $S_{+}$are, respectively, the compressive and extensive strain directions.

$$
\left(1-r^{2}\right)^{1 / 2}>\delta / \sigma
$$

which is always fulfilled when $\delta<0$. Note that the case $\delta^{2} / \sigma^{2}>1$ is a trivial one, for it leads to a growth rate that is unconditionally negative, if $\delta>0$ or positive, if $\delta<0$. In the present case where $\delta / \sigma<1 \mathrm{Eq}$. (6) shows that if non-solenoidal effects are significant and $\delta / \sigma$ is thus close to unity, then $r^{2}$ has to be small enough for the scalar gradient to increase. In other words, $\delta / \sigma$ being given in the range 0 $<\delta / \sigma<1$, the growth rate of the scalar gradient may be negative provided that effective rotation is large enough and thus makes $r^{2}$ assume values near unity. It is only in the special case where $r=0$, for which the scalar gradient aligns with the compressive direction, that the growth rate is unconditionally positive. One can thus infer that: (i) in the non-solenoidal case with $0<\delta / \sigma<1$ it is the effective rotationresulting from vorticity and/or strain axes rotation-which, in spite of prevailing strain, may drive the scalar gradient to decrease and (ii) alignment of the scalar gradient near the extensive direction is a sufficient, but not a necessary condition for the gradient to decrease; a small value of $r^{2}$ indeed implies alignment near to the compressive direction, but $\delta / \sigma$ may be close enough to unity and invalidate the condition defined by Eq. (6).

The latter results can also be understood through a geometric argument in the domain of the gradient orientation variable. From Eq. (5), the norm of the scalar gradient decreases when $\sin \zeta+\delta / \sigma>0$, that is, in terms of angle $\theta$ in the $\left(x_{1}, x_{2}\right)$ system of coordinates,

$$
-\frac{1}{2} \arcsin \left(\frac{\delta}{\sigma}\right)+\theta_{c}+\frac{\pi}{4}<\theta<\frac{1}{2} \arcsin \left(\frac{\delta}{\sigma}\right)+\theta_{c}+\frac{3 \pi}{4},
$$

in which $\theta_{c}$ defines the orientation of the compressive strain axis as $\theta_{c}=-\Phi-\pi / 4$. In the solenoidal case the condition given by Eq. (7) is $\theta_{c}+\pi / 4<\theta<\theta_{c}+3 \pi / 4$ which means that extensive strain acts over a $\pi / 4$-wide angle range on each side of the extensive direction. In the limit where $\delta / \sigma=1$ the condition for the scalar gradient to decrease is 


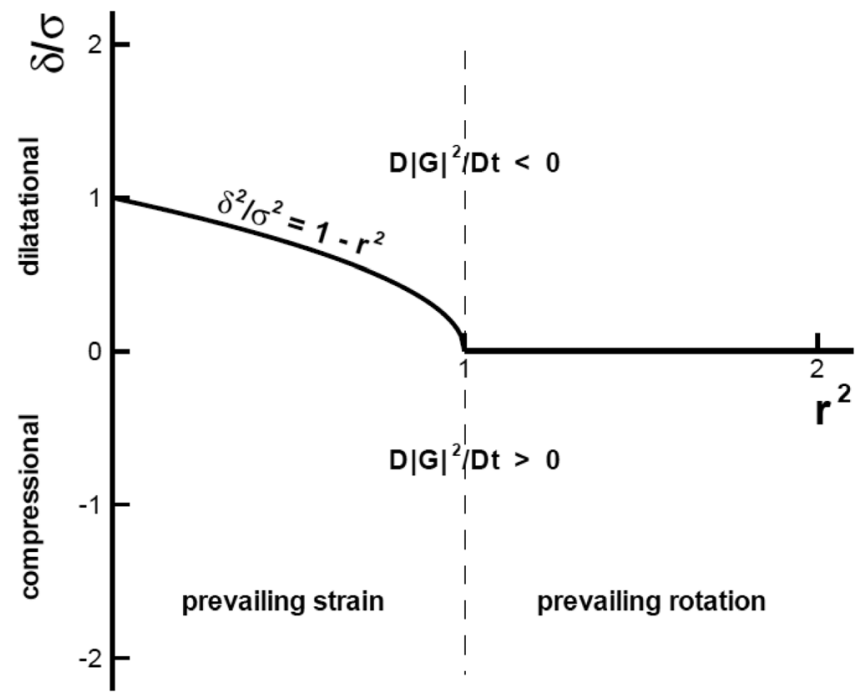

FIG. 2. Sign of the growth rate of the scalar gradient norm in the twodimensional case in function of parameters $r$ and $\delta / \sigma$.

$\theta_{c}<\theta<\theta_{c}+\pi$ showing that, this time, dilatational effects spread over the whole range of orientation angle. For intermediate values- $0<\delta / \sigma<1$ - the orientation domain affected by dilatational effects spreads as $\arcsin (\delta / \sigma)$ with increasing $\delta / \sigma$ (Fig. 1).

When effective rotation prevails over strain $\left(r^{2}>1\right)$ there is no stable fixed point for the scalar gradient orientation and the most probable alignment of the ever-rotating gradient is a bisector of the strain principal axes. ${ }^{24}$ In the solenoidal case the gradient norm varies periodically under the alternate action of compressive and extensive strain, but it neither decreases nor increases on the mean and remains bounded. Non-solenoidal effects, however, make the influence of either compression $(\delta<0)$ or dilatation $(\delta>0)$ spread in the domain of orientation angle promoting, respectively, an overall rise or decay of the gradient norm. The different cases for the sign of the growth rate of the gradient norm are summarized in Fig. 2.

\section{B. Three-dimensional case}

\section{Properties of the strain tensor}

As we work in the strain basis, we just have to consider the properties of the strain eigenvalues. The strain tensor has three real eigenvalues, $\lambda_{i}(i=1,2,3)$, with $\lambda_{1}>\lambda_{2}>\lambda_{3}$ and $\lambda_{1}+\lambda_{2}+\lambda_{3}=\delta$. The eigenvalues of the deviatoric part of the strain tensor are $\lambda_{i}^{\prime}=\lambda_{i}-\delta / 3$ which are obviously such that $\lambda_{1}^{\prime}>\lambda_{2}^{\prime}>\lambda_{3}^{\prime}$ and $\lambda_{1}^{\prime}+\lambda_{2}^{\prime}+\lambda_{3}^{\prime}=0$ with $\lambda_{1}^{\prime}>0, \lambda_{3}^{\prime}<0$ and $\lambda_{2}^{\prime}$ assuming either a positive or a negative value. Then, the three eigenvalues, $\lambda_{i}$, of the strain tensor are negative for $\delta<-3 \lambda_{1}^{\prime}$ and positive for $\delta>-3 \lambda_{3}^{\prime}$; if $-3 \lambda_{1}^{\prime}<\delta<-3 \lambda_{3}^{\prime}$, then there is one positive $\left(\lambda_{1}\right)$ and one negative $\left(\lambda_{3}\right)$ eigenvalues with $\lambda_{2}$ assuming either a positive or a negative value for, respectively, $\delta>-3 \lambda_{2}^{\prime}$ or $\delta<-3 \lambda_{2}^{\prime}$.

\section{Equations for the scalar gradient}

The equations for the scalar gradient in the strain basis in terms of orientation and norm are derived from Eq. (3).

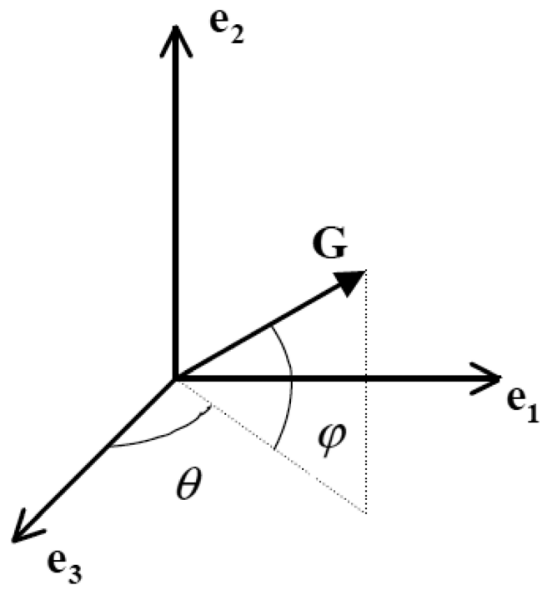

FIG. 3. Spherical coordinates of the scalar gradient, $\boldsymbol{G}$, in the strain basis, $\left(\boldsymbol{e}_{1}, \boldsymbol{e}_{2}, \boldsymbol{e}_{3}\right)$

With the scalar gradient defined in spherical coordinates in the strain basis $\left(\boldsymbol{e}_{1}, \boldsymbol{e}_{2}, \boldsymbol{e}_{3}\right)$ (where the $\boldsymbol{e}_{i}$ 's are the eigenvectors corresponding to eigenvalues $\lambda_{i}$ 's) as $\boldsymbol{G}=|\boldsymbol{G}|$ $(\cos \varphi \sin \theta, \sin \varphi, \cos \varphi \cos \theta)$ (Fig. 3) and following the method used in the incompressible case: ${ }^{28}$

$$
\begin{aligned}
& \frac{D 2 \theta}{D \tau}=\frac{\omega^{\prime}{ }_{2}-\omega^{\prime}{ }_{1} \tan \varphi \sin \theta-\omega^{\prime}{ }_{3} \tan \varphi \cos \theta}{\lambda_{1}^{\prime}-\lambda^{\prime}{ }_{3}}-\sin 2 \theta, \\
& \frac{D 2 \varphi}{D \tau}=\frac{\lambda_{1}^{\prime} \sin ^{2} \theta-\lambda_{2}^{\prime}+\lambda^{\prime}{ }_{3} \cos ^{2} \theta}{\lambda_{1}^{\prime}-\lambda_{3}^{\prime}} \\
& \times\left(\frac{\omega^{\prime}{ }_{3} \sin \theta-\omega_{1}^{\prime} \cos \theta}{\lambda_{1}^{\prime} \sin ^{2} \theta-\lambda_{2}^{\prime}+\lambda_{3}^{\prime} \cos ^{2} \theta}+\sin 2 \varphi\right), \\
& \frac{1}{|\boldsymbol{G}|^{2}} \frac{D|\boldsymbol{G}|^{2}}{D \tau}=\left(\cos 2 \theta+\frac{3 \lambda_{2}^{\prime}}{\lambda_{1}^{\prime}-\lambda_{3}^{\prime}}\right) \cos ^{2} \varphi \\
& -\frac{2}{\lambda_{1}^{\prime}-\lambda_{3}^{\prime}}\left(\lambda_{2}^{\prime}+\delta / 3\right)
\end{aligned}
$$

with $\varphi \neq \pi / 2, D \tau=\left(\lambda_{1}^{\prime}-\lambda_{3}^{\prime}\right) D t$ and $\omega_{i}^{\prime}=\hat{\omega}_{i}-\Omega_{i}$ where the $\hat{\omega}_{i}$ 's and $\Omega_{i}$ 's are, respectively, the components of vorticity and rotation rate of strain principal axes; hatted quantities indicate components in the strain basis. In an inviscid fluid rotation of strain principal axes results from the local action of vorticity and the nonlocal influence of pressure represented by the off-diagonal components of the symmetric part of pressure Hessian, $\boldsymbol{\Pi}_{\mathcal{S}}=1 / 2\left(\boldsymbol{\Pi}+\boldsymbol{\Pi}^{T}\right)$-with $\boldsymbol{\Pi}=\boldsymbol{\nabla}[(1 / \rho) \nabla p]-$, in the strain basis, ${ }^{22}$

$$
\Omega_{i}=-\varepsilon_{\alpha \beta i} \frac{\hat{\omega}_{\alpha} \hat{\omega}_{\beta} / 4+\hat{\Pi}_{\mathcal{S}_{\alpha \beta}}}{\lambda_{\alpha}^{\prime}-\lambda_{\beta}^{\prime}},
$$

in which $\varepsilon_{i j k}$ are the components of the alternating symbol. Note that in the incompressible case the pressure Hessian and its symmetric part coincide.

As compared to the incompressible case, ${ }^{28}$ the orientation equations, Eqs. (8) and (9), remain unchanged which shows that, as in the two-dimensional case (Sec. II A 2), the scalar gradient orientation is not directly affected by non-solenoidal effects. Equation (10) for the gradient norm, by 
contrast, explicitly includes non-solenoidal effects through the velocity divergence, $\delta$. It is to be mentioned again that gradient orientation may indirectly feel non-solenoidal effects through properties of the velocity gradient tensor which are altered by variable mass density.

As already done for incompressible flows, ${ }^{28}$ a simpler though quite realistic problem can be derived from Eqs. (8)(10) by stating that vorticity is aligned with a strain eigenvector. This assumption is physically grounded as it is relevant to small-scale mixing by turbulent flow structures such as strained vortices ${ }^{29}$ and also refers to vortex models approach $^{30,31}$ in which vorticity is considered to be fully aligned with a strain eigenvector. In the incompressible case, as vorticity does tend to align with the "intermediate" strain eigenvector, $\boldsymbol{e}_{2},{ }^{32,33}$ this hypothesis is consistent with turbulence properties. It appears to remain valid in variable-massdensity flows. Statistical alignment of vorticity with the "intermediate" strain eigenvector ${ }^{10}$ and with either the "intermediate" or the extensional eigenvector ${ }^{8}$ — depending on the strain scale that is considered with respect to the vorticity scale-has been confirmed in weakly compressible turbulence. It has also been shown ${ }^{7}$ that in compressible turbulence the alignment of vorticity with the "intermediate" strain eigenvector is weakened by strong dilatation-as vorticity vanishes - but remains significant at moderate dilatation levels. In nonpremixed combustion preferential alignment with $\boldsymbol{e}_{1}$ or $\boldsymbol{e}_{2}$ has been observed in the different flame regions. ${ }^{19-21}$ Furthermore, Boratav et al. ${ }^{21}$ argued that the universality of vorticity alignment properties possibly resulting from the existence of an attracting fixed point of the incompressible Navier-Stokes equations ${ }^{34,35}$ remains valid for variable-density flows.

Now, with the assumption that vorticity is aligned with $\boldsymbol{e}_{2}$ we obviously have $\hat{\omega}_{1}=\hat{\omega}_{3}=0$ which-because of components $\Omega_{1}$ and $\Omega_{3}$ of strain principal axes rotation-is not enough to eliminate $\omega_{1}^{\prime}$ and $\omega_{3}^{\prime}$ from Eqs. (8) and (9). In an Euler, incompressible flow one can turn to account the property according to which: if vorticity is an eigenvector of the strain tensor, then it is an eigenvector of the pressure Hessian tensor. ${ }^{36}$ Interestingly, this property has also been checked in numerical simulations of Navier-Stokes turbulence. ${ }^{37}$ Then, the fact that the strain and the pressure Hessian tensors have one eigenvector in common, say, $\boldsymbol{e}_{i}$, results in $\hat{\Pi}_{i j}=0$ for $j \neq i$ which, with $\hat{\omega}_{j}=0$ for $j \neq i$ and Eq. (11), leads to $\Omega_{j}=0$ for $j \neq i$. For $i=2$, we thus have $\Omega_{1}=\Omega_{3}=0$ and $\omega_{1}^{\prime}=\omega_{3}^{\prime}=0$.

In a variable-mass-density flow the eigenvalue problem is altered by the presence of the baroclinic torque in the vorticity equation and the above property is true only if the baroclinic effect is negligible or in the special case where the baroclinic torque aligns with vorticity (Appendix B). The latter condition is realized when vorticity essentially results from the baroclinic effect which may occur, for instance, at the crossing of a flame front when the unburnt gas flow is irrotational. Making the analytic study of Eqs. (8)-(10) tractable thus needs taking one of the latter hypotheses for granted in addition to assuming vorticity parallel to a strain eigenvector. Then, if vorticity is aligned with $\boldsymbol{e}_{2}, \omega_{1}^{\prime}=\omega_{3}^{\prime}=0$ and Eqs. (8) and (9) simplify as

$$
\begin{gathered}
\frac{D \zeta}{D \tau}=r_{2}-\cos \zeta, \\
\frac{D 2 \varphi}{D \tau}=\frac{1}{2}\left(\sin \zeta-3 \lambda_{2}^{\prime \star}\right) \sin 2 \varphi,
\end{gathered}
$$

while Eq. (10) is rewritten as

$$
\frac{1}{|\boldsymbol{G}|^{2}} \frac{D|\boldsymbol{G}|^{2}}{D \tau}=-\left(\sin \zeta-3 \lambda_{2}^{\prime \star}\right) \cos ^{2} \varphi-2\left(\lambda_{2}^{\prime}{ }^{\star}+\frac{\delta^{\star}}{3}\right),
$$

where $\zeta=2 \theta-\pi / 2, \quad \lambda_{2}^{\prime \star}=\lambda_{2}^{\prime} /\left(\lambda_{1}^{\prime}-\lambda_{3}^{\prime}\right)$ and $\delta^{\star}=\delta /$ $\left(\lambda_{1}^{\prime}-\lambda_{3}^{\prime}\right)$. Parameter $r_{2}$ represents strain persistence and measures the influence of rotation with respect to strain as $r_{2}=\omega_{2}^{\prime} /\left(\lambda_{1}^{\prime}-\lambda_{3}^{\prime}\right)$.

\section{Kinematics of the scalar gradient}

The equations for the scalar gradient orientation, Eqs. (12) and (13), keep the same form they have in the incompressible case and the original analysis ${ }^{28}$ of fixed points and of the orientation dynamics in terms of $r_{2}$ and $\lambda_{2}^{\prime \star}-$ confirmed by a stochastic Lagrangian model ${ }^{38}$-remains unchanged. In this regard, the special value of the azimuthal angle, $\varphi=0$, is a stable fixed point of Eq. (13) if $r_{2}^{2} \leq 1$ and $\left(1-r_{2}^{2}\right)^{1 / 2}+3 \lambda_{2}^{\prime \star}>0$ or $r_{2}^{2}>1$ and $\lambda_{2}^{\prime \star}>0$. In these conditions, as the scalar gradient tends to align in the $\left(\boldsymbol{e}_{1}, \boldsymbol{e}_{3}\right)$ plane, Eq. (14) for the gradient norm is

$$
\frac{1}{|\boldsymbol{G}|^{2}} \frac{D|\boldsymbol{G}|^{2}}{D \tau}=-\sin \zeta+\lambda_{2}^{\prime \star}-\frac{2}{3} \delta^{\star} .
$$

As mentioned in Sec. II B 1 , if $\delta^{\star}<-3 \lambda_{1}^{\prime \star}$, then the strain tensor has three negative eigenvalues which necessarily brings about a positive growth rate of the gradient norm; in this case it is indeed easy to show that $\lambda_{2}^{\prime \star}-2 \delta^{\star} / 3>1$ which makes the right-hand side of Eq. (15) positive. If $\delta^{\star}>-3 \lambda_{3}^{\prime \star}$ (the eigenvalues of the strain tensor are positive), then $\lambda_{2}^{\prime \star}-2 \delta^{\star} / 3<-1$ and the norm growth rate is negative.

The intermediate case, $-3 \lambda_{1}^{\prime \star}<\delta^{\star}<-3 \lambda_{3}^{\prime \star}$ (i.e., $\left.-1<\lambda_{2}^{\prime \star}-2 \delta^{\star} / 3<1\right)$, in which the strain tensor has one positive $\left(\lambda_{1}\right)$ and one negative $\left(\lambda_{3}\right)$ eigenvalues is more complex. If strain prevails $\left(r_{2}{ }^{2} \leq 1\right)$, then angle $\zeta$ tends to the fixed point, $\zeta_{e q}=-\arccos \left(r_{2}\right),{ }^{28}$ and Eq. (15) can be written as

$$
\frac{1}{|\boldsymbol{G}|^{2}} \frac{D|\boldsymbol{G}|^{2}}{D \tau}=\left(1-r_{2}^{2}\right)^{1 / 2}+\lambda_{2}^{\prime \star}-\frac{2}{3} \delta^{\star}
$$

The growth rate of the scalar gradient norm is positive provided that

$$
\left(1-r_{2}^{2}\right)^{1 / 2}>\frac{2}{3} \delta^{\star}-\lambda_{2}^{\prime \star}
$$

which is to be compared to Eq. (6) in the two-dimensional case. The condition defined in Eq. (17) is specially realized for $\delta^{\star}<3 \lambda_{2}^{\prime \star} / 2$ and more specifically for $\lambda_{2}^{\prime \star}>0$ and $\delta^{\star}<0$, in other words, when non-solenoidal compressional effects are combined with stretching of vorticity by the 
deviatoric part of the strain tensor. If $\delta^{\star}>3 \lambda_{2}^{\prime *} / 2$, then the condition for a positive norm growth rate is not always realized even if $\delta^{\star}<0$ which distinguishes the three- from the two-dimensional case (Sec. II A 3). More generally, Eq. (17) shows that $2 \delta^{\star} / 3-\lambda_{2}^{\prime \star}$ being given, effective rotationwhich increases $r_{2}^{2}$-tends to oppose positive values of the norm growth rate. It is only strongly dominating strain $\left(r_{2} \simeq 0\right)$ that makes the growth rate of the scalar gradient norm unconditionally positive. As in the two-dimensional case (Sec. II A 3), in this three-dimensional situation, where vorticity aligns with a strain eigenvector it is effective rotation that may drive the scalar gradient to decrease, in spite of prevailing strain; again, alignment of the scalar gradient with the extensive direction of strain does not appear as a necessary condition for the gradient to decrease.

If effective rotation prevails $\left(r_{2}^{2}>1\right)$ and $\lambda_{2}^{\prime *}$ is positive, then $\varphi=0$ is a stable fixed point of Eq. (13), but Eq. (12) has no fixed point and the scalar gradient rotates in the plane $\left(\boldsymbol{e}_{1}\right.$, $\left.\boldsymbol{e}_{3}\right)$. In the intermediate case, $-1<\lambda_{2}^{\prime \star}-2 \delta^{\star} / 3<1$, the scalar gradient norm fluctuates while increasing on the mean if $2 \delta^{\star} / 3<\lambda_{2}^{\prime \star}$. This behavior is obviously promoted by compressional effects $\left(\delta^{\star}<0\right)$, but is not inconsistent with dilatation $\left(\delta^{\star}>0\right)$ provided that $\lambda_{2}^{\prime \star}$ is large enough. If $2 \delta^{\star} / 3>\lambda_{2}^{\prime \star}$-which obviously implies $\delta^{\star}>0$ - then the gradient norm fluctuates while decreasing on the mean. The

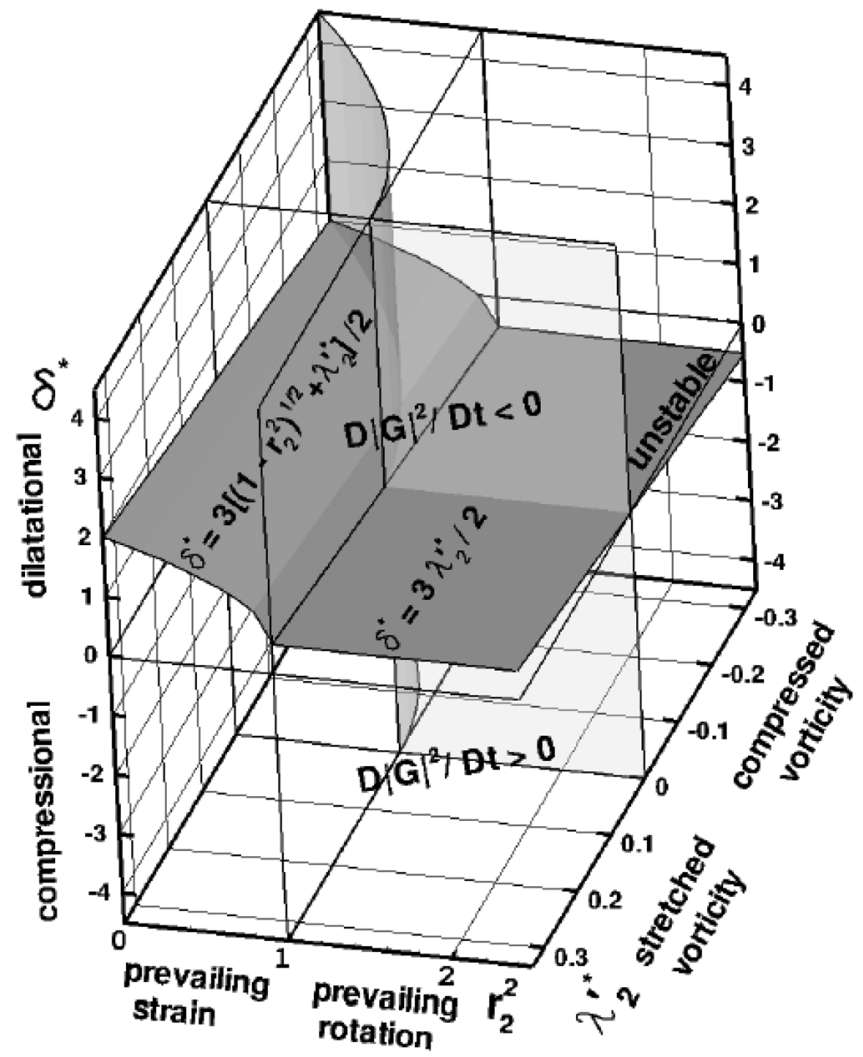

FIG. 4. Sign of the growth rate of the scalar gradient norm in the threedimensional case in function of parameters $\lambda_{2}{ }^{\star \star}, r_{2}$, and $\delta^{\star}$ in the case where vorticity aligns with the "intermediate" strain direction; the shaded surface separates the positive from the negative growth rates; the fixed point of the orientation equation is stable for $r_{2}{ }^{2} \leq 1$ and $\lambda_{2}{ }^{1 \star}>-\left(1-r_{2}{ }^{2}\right)^{1 / 2} / 3$ or $r_{2}^{2}>1$ and $\lambda_{2}^{\prime \star}>0$; parameters $r_{2}$ and $\delta^{\star}$ are unbounded and $-1 / 3 \leq \lambda_{2}^{\prime \star} \leq 1 / 3$. sign of the growth rate of the scalar gradient norm in function of parameters $\lambda_{2}^{\prime \star}, r_{2}$, and $\delta^{\star}$ is given in Fig. 4.

Finally, as in the two-dimensional case (Sec. II A 3), the effects of increasing $\left|\delta^{\star}\right|$ upon the scalar gradient norm may be seen as resulting from the spreading over the orientation angle domain of the corresponding non-solenoidal-compressional or dilatational-influence. As an example, we consider Eq. (15) with $\lambda_{2}^{\prime \star}>0$ which strengthens the rise of the scalar gradient in the solenoidal case $\delta^{\star}=0$ and check the result of increasing dilatation through $\delta^{\star}>0$. We restrict to $-1 \leq \lambda_{2}^{\prime \star}-2 \delta^{\star} / 3 \leq 1 \quad\left(\lambda_{2}^{\prime \star}-2 \delta^{\star} / 3<-1 \quad\right.$ or $\lambda_{2}^{\prime \star}-2 \delta^{\star} / 3>1$ resulting in a trivial, unconditional decay or growth of the gradient norm respectively). For $\lambda_{2}^{\prime \star}-2 \delta^{\star} / 3 \leq 0$, $\delta^{\star}$ is bounded as $3 \lambda_{2}^{\prime \star} / 2 \leq \delta^{\star} \leq 3\left(1+\lambda_{2}{ }^{\prime \star}\right) / 2$. The norm of the scalar gradient decreases for negative values of the righthand side of Eq. (15) which is expressed by

$$
\begin{aligned}
& \frac{1}{2} \arcsin \left(\lambda_{2}^{\prime}{ }^{\star}-\frac{2}{3} \delta^{\star}\right)+\frac{\pi}{4}<\theta<-\frac{1}{2} \arcsin \left(\lambda_{2}^{\prime}{ }^{\star}-\frac{2}{3} \delta^{\star}\right) \\
& \quad+\frac{3 \pi}{4} .
\end{aligned}
$$

This condition implies that for the minimal value of $\delta^{\star}$, $\delta^{\star}=3 \lambda_{2}^{\prime \star} / 2$, decrease of the gradient norm occurs for the angle range $\pi / 4<\theta<3 \pi / 4$ in the $\left(\boldsymbol{e}_{1}, \boldsymbol{e}_{3}\right)$ plane which exactly corresponds to the influence domain of extensive strain parallel to $\boldsymbol{e}_{1}$ when there is no straining in $\boldsymbol{e}_{2}$ direction, while for the maximal value, $\delta^{\star}=3\left(1+\lambda_{2}^{\prime \star}\right) / 2$, the whole angle range, $0<\theta<\pi$, is affected by dilatation; the latter case corresponds to dominating non-solenoidal, dilatational effect, while the former expresses the balance between the straining effect promoting the scalar gradient and the dilatational effect that tends to decrease the gradient norm. Intermediate, increasing values of $\delta^{\star}$ make the dilatationalinfluenced orientation domain spread in the $\left(\boldsymbol{e}_{1}, \boldsymbol{e}_{3}\right)$ plane. In the case where $\lambda_{2}^{\prime \star}-2 \delta^{\star} / 3 \geq 0$ a similar analysis shows that the non-solenoidal, dilatational effect at most balances the compressive straining in the $\left(\boldsymbol{e}_{1}, \boldsymbol{e}_{3}\right)$ plane. The kinematics of the scalar gradient in the case where vorticity aligns with eigenvector $\boldsymbol{e}_{1}$ is summarized in Appendix $\mathrm{C}$ and the sign of the growth rate of the gradient norm in function of parameters $\lambda_{1}{ }^{\star \star}, r_{1}$, and $\delta^{\star}$ is given in Fig. 5 .

\section{DISCUSSION ON NON-SOLENOIDAL KINEMATICS OF SCALAR GRADIENT IN PREMIXED FLAMES}

\section{A. Recent studies on heat release effects in premixed flames}

In flames large spatial variations of mass density resulting from heat release deeply alter the properties of the velocity gradient which, in turn, may bring non-solenoidal effects in the kinematics of scalar gradient. Negative stretching of scalar gradient $\left(-G_{\alpha \alpha} S_{\alpha \beta} G_{\beta}<0\right)$ causing the lessening of production of scalar dissipation rate-and hence a possibly reduced efficiency of micromixing-is a striking outcome of non-solenoidal effects. This feature has been emphasized for the gradient of a reacting scalar in turbulent premixed flames and shown to be specially significant when a large Damköhler number, Da, is combined with a small Karlovitz number, 


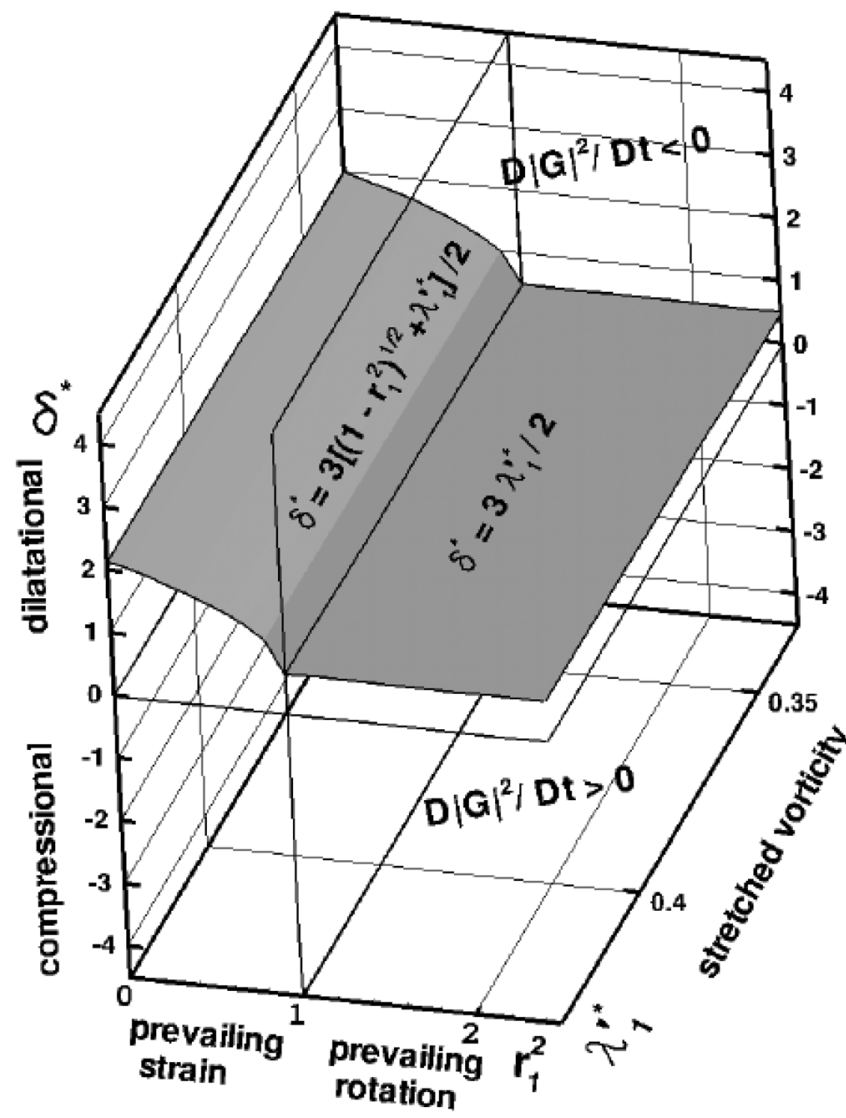

FIG. 5. Sign of the growth rate of the scalar gradient norm in the threedimensional case in function of parameters $\lambda_{1}{ }^{\star \star}, r_{1}$, and $\delta^{\star}$ in the case where vorticity aligns with the extensive strain direction; the shaded surface separates the positive from the negative growth rates; parameters $r_{1}$ and $\delta^{\star}$ are unbounded and $\lambda_{1}{ }^{\prime \star} \geq 1 / 3$.

Ka. ${ }^{15-17}$ The latter measures the competing effects of chemical and straining mechanisms, $\mathrm{Ka}=\left(L_{D} / S_{f}\right) \tau_{\eta}{ }^{-1}$, while the former compares the chemical process to the large-scale turbulent transport, $\mathrm{Da}=\left(S_{f} / L_{D}\right) \tau_{t},{ }^{39} S_{f}$ and $L_{D}$ are, respectively, the laminar flame velocity and the laminar flame thickness and $\tau_{\eta}$ and $\tau_{t}$ the Kolmogorov and the integral time scales. Ratio $L_{D} / S_{f}$ defines the time scale of the chemical process.

Mura et al. ${ }^{16}$ have shown the dependence of velocity gradient and reacting scalar gradient interaction on the thermal expansion coefficient, $\gamma=\rho_{u} / \rho_{b}$, where $\rho_{u}$ and $\rho_{b}$ are, respectively, the mass densities in the unburnt and burnt gases. The study by Chakraborty and Swaminathan ${ }^{17}$ also suggests an influence of the expansion ratio as negative stretching of scalar gradient may be observed in a flame with $\mathrm{Da}<1$ and $\mathrm{Ka}>1$ in regions where heat release is large. The latter work ascribes negative stretching to alignment of scalar gradient with the extensive direction of strain as originally proposed for flames with $\mathrm{Da}>1$ and $\mathrm{Ka}<1 .{ }^{15}$ Negative stretching has also been found in flames with $\mathrm{Da}>1$ and $\mathrm{Ka}>1$ and interpreted, too, through alignment with extensive strain. ${ }^{18}$

\section{B. The infinitely thin flame limit}

An indirect proof of non-solenoidal effects upon scalar gradient stretching in flames is given by the case in which a premixed flame is regarded as a discontinuity separating two regions with different constant densities. In this problem where the flow on each side of the flame front is treated assuming incompressibility the growth rate of the norm of the passive scalar gradient is not found to take negative values. ${ }^{40}$ Nevertheless, rotation of strain principal axes caused by anisotropy of the pressure Hessian and vorticity produced at the flame front result in an effective rotation which plays on scalar gradient kinematics through alignment properties. ${ }^{40}$

The effect of the discontinuity in mass density upon the orientation of the strain principal axes is to be specially emphasized. As described in a previous study, ${ }^{40}$ the flame front is assumed to be stabilized at distance $x=d$ in a stagnation flow. In the incoming unburnt flow $(x>d), \sigma_{11}=-\sigma_{0} / 2$ and $\sigma_{12}=0$; past the interface the normal component of strain is conserved, $\sigma_{11}\left(d^{-}\right)=-\sigma_{0} / 2$, while the shear component experiences a discontinuity such that $\sigma_{12}\left(d^{-}\right)$ $=-\sigma_{0}(\gamma-1)^{1 / 2} \arctan \left[(\gamma-1)^{1 / 2}\right] y / 4 d$ which makes the orientation of the strain principal axes change from $\Phi\left(d^{+}\right)=-\pi / 4$ on the unburnt side to

$$
\begin{aligned}
\Phi\left(d^{-}\right)=- & \pi / 4-\left[\pi / 2-\arctan \left[\left[(\gamma-1)^{1 / 2}\right.\right.\right. \\
& \left.\left.\left.\times \arctan \left[(\gamma-1)^{1 / 2}\right] y / 4 d\right]^{-1}\right]\right] / 2,
\end{aligned}
$$

on the burnt side, where $y$ is the ordinate defined along the flame front and $d^{+}$and $d^{-}$are infinitesimal distances from the front on the unburnt and burnt sides, respectively. The discontinuity thus makes the orientation of the compressive direction tilt from $\theta_{c}\left(d^{+}\right)=0$ in the unburnt region to $\theta_{c}\left(d^{-}\right)$ $=-\Phi\left(d^{-}\right)-\pi / 4$ in the burnt region. For small values of the thermal expansion coefficient, $\gamma \simeq 1, \theta_{c}\left(d^{-}\right) \simeq 0$ which means that there is no tilting of the compressive direction; for large values, $\gamma \gg 1, \theta_{c}\left(d^{-}\right)=\pi / 4$. The compressive eigenvector spans the angular range $[0, \pi / 4]$ as $\gamma$ is increased (Fig. 6). As we consider a non-reacting scalar, the orientation

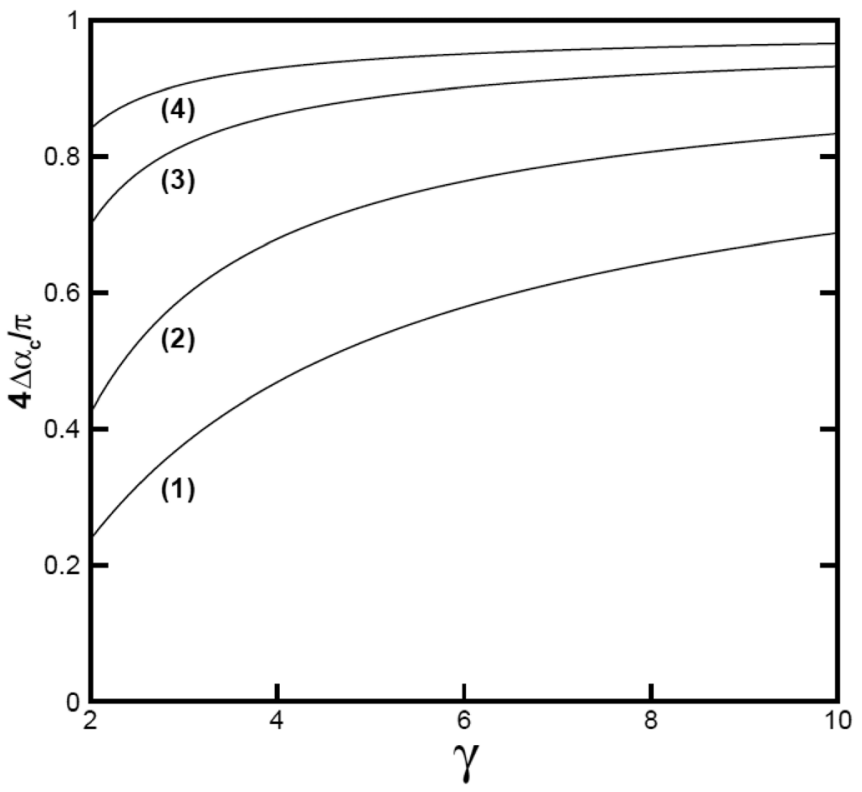

FIG. 6. Tilting of strain principal axes, $\Delta \theta_{c}=\theta_{c}\left(d^{-}\right)-\theta_{c}\left(d^{+}\right)$, normalized by the maximum, $\pi / 4$-tilting, plotted in function of the thermal expansion coefficient; (1): $y / d=1 ;(2): y / d=2 ;(3): y / d=5 ;(4): y / d=10$. 


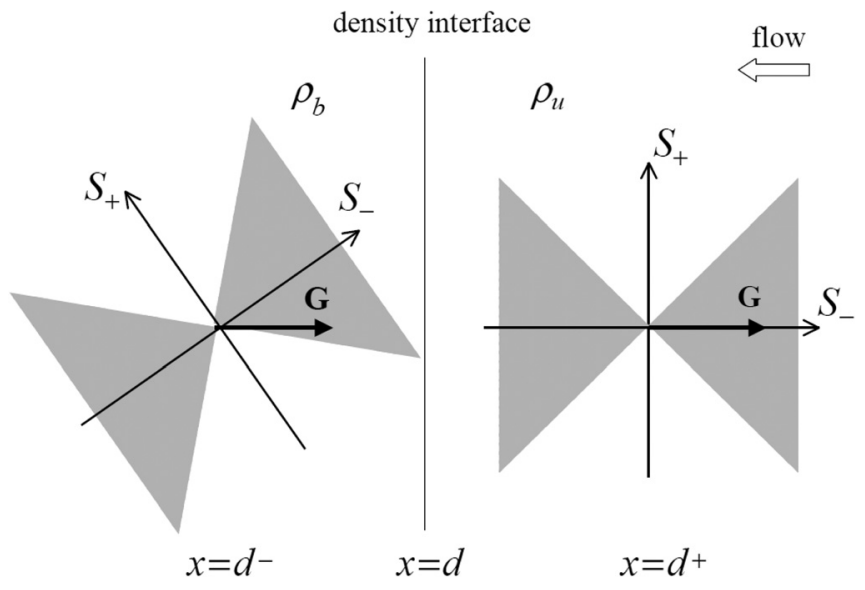

FIG. 7. Tilting of strain principal axes for large values of the thermal expansion coefficient $(\gamma \gg 1)$ in the case where the flame front is considered as an interface of mass density; $S_{-}$and $S_{+}$are, respectively, the compressive and extensive strain directions; strain is compressive inside the shaded area and extensive outside.

of gradient $\mathbf{G}$ could be immaterial, but consistently with the reacting problem $\mathbf{G}\left(d^{+}\right)$has to be assumed initially normal to the front as shown in Fig. 7. The scalar gradient does not respond to the infinitely fast change in the properties of the velocity gradient tensor resulting from the density interface and $\mathbf{G}\left(d^{-}\right)$keeps the initial orientation. Clearly, even for large values of the expansion coefficient, the maximum, $\pi / 4$-tilting of strain principal axes is not enough to make the scalar gradient fall into the extensive-strain-influenced region (Fig. 7) and to bring about a negative stretching. However, misalignment of $\boldsymbol{G}$ with respect to the compressional direction resulting from tilting of strain principal axes may cause a drop in the gradient growth rate. It is likely that in a finite flame front non-solenoidal effects combine with this kind of tilting mechanism-smoother though it may be.

\section{Non-solenoidal effects}

Regarding the variable-mass-density effects encountered in a finite-length flame front, we restrict to the two-dimensional case and ground the discussion on the analysis of Sec. II. Now, from the latter, $\delta / \sigma$ appears as the main parameter governing the non-solenoidal kinematics of the scalar gradient. A rough estimate of $\delta$ for a plane, stabilized flame can be derived as $\delta \simeq-u / \rho \cdot d \rho / d x$ - where $u$ is the velocity of the stabilizing flow-, that is, $\delta \simeq S_{f} / L_{D} \cdot(\gamma-1) / \gamma$. Ratio $(\gamma-1) / \gamma$ is the order of unity for standard values of $\gamma$, namely $\gamma \sim 5-6$. It follows that $\delta / \sigma \propto\left(S_{f} / L_{D}\right) \sigma^{-1}=\mathrm{Ka}^{-1}$. Significant non-solenoidal effects resulting from heat release via chemical reactions are thus expected for small values of the Karlovitz number corresponding to low strain and/or fast chemical process. A more refined derivation of $\delta$ assuming, for instance, an exponential profile of mass density across the preheat zone of the flame leads to $\delta / \sigma=f\left(x / L_{D}, \gamma\right) \mathrm{Ka}^{-1}$ where $f$ is a function of $\gamma$ and of the nondimensional distance, $x / L_{D}$, across the flame. Function $f$-as well as ratio $(\gamma-1) / \gamma$-is a monotonous, increasing function of $\gamma$ thus suggesting a possible enhancement of the influence of the Karlovitz number by heat release. ${ }^{16,17}$
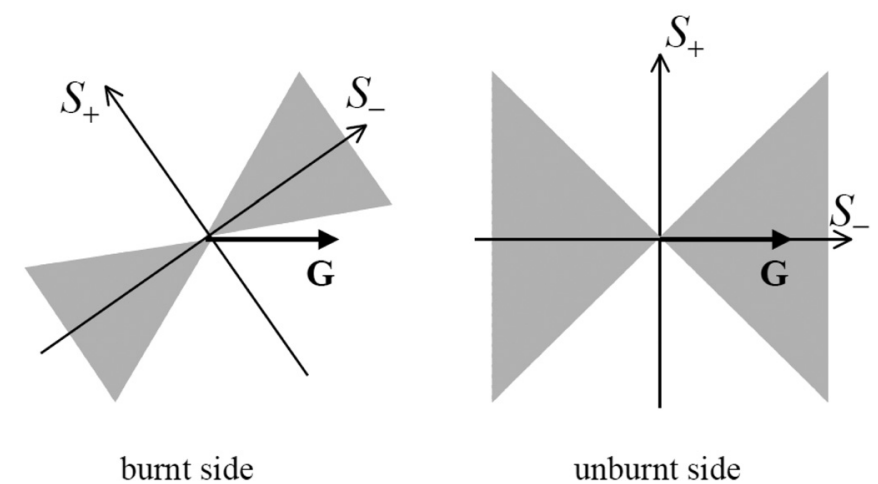

unburnt side

FIG. 8. Combination of tilting of strain principal axes with extensive strain resulting from non-solenoidal effects for $0<\delta / \sigma<1 ; S_{-}$and $S_{+}$are, respectively, the compressive and extensive strain directions; strain is compressive inside the shaded area and extensive outside.

Negative stretching of the scalar gradient is most likely to occur in the case of very small Karlovitz number-hence large $\delta / \sigma-$. As shown in Sec. II, when $\delta / \sigma>1$ the eigenvalues of strain are positive and the growth rate of the scalar gradient is unconditionally negative (see Fig. 2). Note, too, that in nonpremixed flames Nomura and Elghobashi ${ }^{19}$ mentioned a possible shift of strain eigenvalues p.d.f's toward positive values as a result of non-solenoidal effect. In the case of moderate Karlovitz number, which would correspond to $0<\delta / \sigma<1$, the scalar gradient may experience a negative stretching in spite of the existence of a compressive direction of strain (Sec. II A 3). However, in the view where the scalar gradient is assumed to be aligned with the compressive direction on the unburnt side of the flame the mechanism described in Sec. II A 3 (i.e., the spreading of the extensive effect of strain over the orientation angle domain) is not enough to cause a negative stretching unless the strain principal axes rotate; as long as $0<\delta / \sigma<1$, a compressiondominated range of orientation angle remains. But the combination of extensive strain due to non-solenoidal effects with rotation of strain principal axes may drive the scalar gradient to fall into the extensive-strain-influenced angle range and to undergo a negative stretching (Fig. 8). This picture results from an idealized model of the premixed flame and would deserved to be checked for more realistic modelled or simulated flames.

\section{CONCLUSION}

Analysis of the equations for the orientation and norm of the gradient of a passive scalar in two- and three-dimensional flows with variable mass density reveals interesting features regarding the kinematics of the scalar gradient experiencing non-solenoidal effects through the action of the velocity gradient.

While non-solenoidal effects do not directly alter the equation for the orientation of the scalar gradient, they are explicitly included in the equation for the norm through velocity divergence. More specifically, in two-dimensional flows both the strain persistence parameter, $r$, and the ratio, 
$\delta / \sigma$, of velocity divergence to the norm of the deviatoric part of the strain tensor govern the evolution of the scalar gradient. In the compressional case $(\delta / \sigma<0)$, the scalar gradient is unconditionally enhanced. The dilatational case $(\delta / \sigma>0)$ involves a more complex behavior. Large dilatation $(\delta / \sigma>1)$ unconditionally lessens the scalar gradient, but moderate dilatation $(0<\delta / \sigma<1)$ has to be combined with effective rotation $(r \neq 0)$ to make the scalar gradient norm decrease which may occur even for prevailing strain $\left(r^{2}<1\right)$. The latter result is explained by two facts: (i) rotation makes the equilibrium orientation of the scalar gradient draw away from the compressional direction and (ii) the orientation range affected by extensive strain is increased by dilatation. Alignment with the extensive direction of strain is thus a sufficient, but not a necessary condition for a negative growth rate—or "stretching"—of the scalar gradient.

In three-dimensional flows the general equations for the scalar gradient also show that only the equation for the gradient norm is affected by non-solenoidal effects through the velocity divergence. Analytic study is untractable unless vorticity is assumed to be aligned with a strain principal axis. In addition, it has to be restricted to weak baroclinic effects or to the special case where vorticity is parallel to the baroclinic torque. The latter is not unrealistic and may be reminiscent of flow conditions in which vorticity is essentially produced through the baroclinic effect, a situation that may even be viewed as a local one. The picture derived from the analysis needs a strain persistence parameter, the velocity divergence and the eigenvalue of the deviatoric part of the strain tensor corresponding to the eigenvector parallel to vorticity. Large compressional $\left(\delta<-3 \lambda_{1}{ }^{\prime}\right)$ or dilatational $\left(\delta>-3 \lambda_{3}{ }^{\prime}\right)$ nonsolenoidal effects unconditionally result in either growth or decay of the scalar gradient norm. For moderate non-solenoidal effects $\left(-3 \lambda_{1}{ }^{\prime}<\delta<-3 \lambda_{3}{ }^{\prime}\right)$ one has to distinguish between prevailing strain or prevailing effective rotation via the strain persistence parameter. As in two-dimensional flows, effective rotation promotes negative norm growth rate in the case of non-solenoidal, dilatational effects. It also appears that the scalar gradient does not need to align with the extensive strain direction to experience a negative growth rate of its norm.

A tentative discussion of the case of reacting flows with heat release has been drawn from the two-dimensional analysis. In premixed flames parameter $\delta / \sigma$ comes to the reciprocal of the Karlovitz number. Significant variable-massdensity effects experienced by scalar gradients through the action of velocity gradient are thus expected for small values of the Karlovitz number. For moderate Karlovitz number, dilatational effects resulting from heat release may combine with other mechanisms such as tilting of strain principal axes to bring about the lessening of the scalar gradient.

\section{APPENDIX A: NON-ZERO MEAN SCALAR GRADIENT IN THE TWO-DIMENSIONAL CASE}

In the presence of a mean scalar gradient, $\langle\boldsymbol{G}\rangle$, the concern is usually on the stretching of the fluctuating gradient, $\boldsymbol{G}^{\prime}=\boldsymbol{G}-\langle\boldsymbol{G}\rangle .{ }^{15-17}$ With the method used in Sec. II A 2, the equations for $\boldsymbol{G}^{\prime}$ in terms of orientation, $\xi$, and norm, $\left|\boldsymbol{G}^{\prime}\right|$, are

$$
\begin{aligned}
& \frac{D \xi}{D \tau}=r-\cos \xi+ \frac{|\langle\boldsymbol{G}\rangle|}{\left|\boldsymbol{G}^{\prime}\right|}\left\{\frac{\omega}{\sigma} \cos \left[\frac{1}{2}\left(\xi-\zeta_{\langle\boldsymbol{G}\rangle}\right)\right]\right. \\
&\left.-\cos \left[\frac{1}{2}\left(\xi+\zeta_{\langle\boldsymbol{G}\rangle}\right)\right]+\frac{\delta}{\sigma} \sin \left[\frac{1}{2}\left(\xi-\zeta_{\langle\boldsymbol{G}\rangle}\right)\right]\right\} \\
& \frac{1}{\left|\boldsymbol{G}^{\prime}\right|^{2}} \frac{D\left|\boldsymbol{G}^{\prime}\right|^{2}}{D \tau}=-\left(\sin \xi+\frac{\delta}{\sigma}\right) \\
&-\frac{|\langle\boldsymbol{G}\rangle|}{\left|\boldsymbol{G}^{\prime}\right|}\left\{\sin \left[\frac{1}{2}\left(\xi+\zeta_{\langle\boldsymbol{G}\rangle}\right)\right]\right. \\
&-\frac{\omega}{\sigma} \sin \left[\frac{1}{2}\left(\xi-\zeta_{\langle\boldsymbol{G}\rangle}\right)\right] \\
&\left.+\frac{\delta}{\sigma} \cos \left[\frac{1}{2}\left(\xi-\zeta_{\langle\boldsymbol{G}\rangle}\right)\right]\right\}
\end{aligned}
$$

in which $\zeta_{\langle G\rangle}$ is the orientation variable of the mean scalar gradient.

Unless large fluctuating gradients such that $|\boldsymbol{G}|^{\prime} \gg|\langle\boldsymbol{G}\rangle|$ are considered, the presence of a mean gradient makes the equations for the fluctuating gradient more complex than Eqs. (4) and (5) for the instantaneous gradient and involves the following new features:

(i) the study of fixed points for the orientation of the fluctuating gradient cannot be made analytically from Eq. (A1) as Lapeyre et al $^{24}$ did in the simpler case in which there is no mean scalar gradient;

(ii) the respective kinematics of the fluctuating and instantaneous gradients are different. In particular, as shown by the additional terms in Eq. (A2), even in the solenoidal case where $\delta=0$ alignment of $\boldsymbol{G}^{\prime}$ with either the compressive $(\xi=-\pi / 2)$ or extensive $(\xi=\pi / 2)$ direction may not involve, respectively, the rise or decrease of the norm of the fluctuating gradient;

(iii) the presence of the mean gradient makes new mechanisms come into play. As shown by Eqs. (A1) and (A2), through the relative orientations of the fluctuating and mean gradients the orientation of the fluctuating gradient is influenced by its norm and is also directly affected by non-solenoidal effects; vorticity directly plays on the norm of the fluctuating gradient.

It is only in the special case where $\boldsymbol{G}^{\prime}$ and $\langle\boldsymbol{G}\rangle$ are precisely aligned — or anti-aligned — that a more standard kinematics of the fluctuating gradient is retrieved.

\section{APPENDIX B: EIGENVALUE PROBLEM IN THE NON-SOLENOIDAL CASE}

In the non-solenoidal case the equations for the strain tensor and vorticity components are

$$
\begin{gathered}
\frac{D S_{i j}}{D t}=-S_{i \alpha} S_{\alpha j}-\frac{1}{4}\left(\omega_{i} \omega_{j}-\omega_{\alpha} \omega_{\alpha} \delta_{i j}\right)-\Pi_{\mathcal{S}_{i j}}, \\
\frac{D \omega_{i}}{D t}=S_{i \alpha} \omega_{\alpha}+B_{i} .
\end{gathered}
$$

In Eq. (B2) $B_{i}=-\varepsilon_{i \alpha \beta} \Pi_{\alpha \beta}$. This term represents the components of the anti-symmetric part of $\boldsymbol{\Pi}$ and is also nothing but the $i^{t h}$ component of the baroclinic torque, $\left(1 / \rho^{2}\right) \nabla \rho \times \nabla p$. 
Now, if vorticity is an eigenvector of the strain tensor, then $S_{i \alpha} \omega_{\alpha}=\lambda \omega_{i}$ and, with Eq. (B2)

$$
\frac{D \omega_{i}}{D t}=\lambda \omega_{i}+B_{i},
$$

and the product of each side of Eq. (B3) by $\boldsymbol{S}$ gives

$$
S_{i \beta} \frac{D \omega_{\beta}}{D t}=\lambda^{2} \omega_{i}+S_{i \beta} B_{\beta} .
$$

Using Eqs. (B1) and (B2) in Eq. (B4) leads to

$$
\Pi_{\mathcal{S}_{i \beta}} \omega_{\beta}=-\left(\lambda^{2}+\frac{D \lambda}{D t}\right) \omega_{i}+\left(S_{i \beta}-\lambda \delta_{i \beta}\right) B_{\beta} .
$$

As compared to the incompressible case, ${ }^{36}$ Eq. (B5) includes an additional term resulting from the baroclinic effect. Provided that the latter effect is negligible, Eq. (B5) defines $\omega$ as an eigenvector of $\boldsymbol{\Pi}_{\mathcal{S}}$. The last term of Eq. (B5) is exactly zero in the special case where the baroclinic torque is aligned with vorticity and is thereby an eigenvector of $\boldsymbol{S}$.

\section{APPENDIX C: KINEMATICS OF SCALAR GRADIENT FOR VORTICITY ALIGNING WITH THE EXTENSIVE STRAIN DIRECTION}

In this case it is convenient to define the scalar gradient in spherical coordinates as $\boldsymbol{G}=|\boldsymbol{G}|(\sin \varphi, \cos \varphi \sin \theta$, $\cos \varphi \cos \theta)$ where, this time, $\varphi$ is the azimuthal angle from the $\left(\boldsymbol{e}_{2}, \boldsymbol{e}_{3}\right)$ plane to vector $\boldsymbol{G}$ and $\theta$ is the angle between eigenvector $\boldsymbol{e}_{3}$ and the projection of $\boldsymbol{G}$ in the $\left(\boldsymbol{e}_{2}, \boldsymbol{e}_{3}\right)$ plane. The equations for the orientation and norm of $\boldsymbol{G}$ can be derived by permuting $\lambda_{1}{ }^{\prime \star}$ and $\lambda_{2}{ }^{\prime \star}$ in Eqs. (12)-(14),

$$
\begin{gathered}
\frac{D \zeta}{D \tau}=r_{1}-\cos \zeta, \\
\frac{D 2 \varphi}{D \tau}=\frac{1}{2}\left(\sin \zeta-3 \lambda_{1}^{\prime \star}\right) \sin 2 \varphi \\
\frac{1}{|\boldsymbol{G}|^{2}} \frac{D|\boldsymbol{G}|^{2}}{D \tau}=-\left(\sin \zeta-3 \lambda_{1}^{\prime \star}\right) \cos ^{2} \varphi-2\left(\lambda_{1}^{\prime \star}+\frac{\delta^{\star}}{3}\right),
\end{gathered}
$$

with $\zeta=2 \theta-\pi / 2, D \tau=\left(\lambda_{2}^{\prime}-\lambda_{3}^{\prime}\right) D t, \lambda_{1}^{\prime \star}=\lambda_{1}^{\prime} /\left(\lambda_{2}^{\prime}-\lambda_{3}^{\prime}\right)$, $\delta^{\star}=\delta /\left(\lambda_{2}^{\prime}-\lambda_{3}^{\prime}\right)$ and $r_{1}=-\omega_{1}^{\prime} /\left(\lambda_{2}^{\prime}-\lambda_{3}^{\prime}\right)$.

This case is simpler than the situation in which vorticity is assumed to align with $\boldsymbol{e}_{2}$ because $\lambda_{1}^{\prime *}$ is essentially positive and $\varphi=0$ is an unconditionally stable fixed point of Eq. (C2). For $r_{1}^{2} \leq 1$ Eq. (C1) has a stable fixed point given by $\zeta_{e q}=-\arccos \left(r_{1}\right)$, while for $r_{1}^{2}>1 \mathrm{Eq}$. (C1) has no stable fixed point and $\boldsymbol{G}$ rotates in the $\left(\boldsymbol{e}_{2}, \boldsymbol{e}_{3}\right)$ plane. $^{28}$

Regarding the evolution of the gradient norm for $\varphi=0$, cases $\delta^{\star}<-3 \lambda_{1}{ }^{\prime \star}$ and $\delta^{\star}>-3 \lambda_{3}{ }^{\star \star}$ are trivial (Sec. II B 3) and, respectively, correspond to a positive or a negative growth rate. For intermediate values of $\delta^{\star},-3 \lambda_{1}{ }^{\star \star}<\delta^{\star}$ $<-3 \lambda_{3}{ }^{\text {'* }}$ (which corresponds to $-1<\lambda_{1}{ }^{1 \star}-2 \delta^{\star} / 3<1$ ), one has to distinguish $r_{1}^{2} \leq 1$ from $r_{1}^{2}>1$. In the latter case the norm of the scalar gradient fluctuates while increasing or decreasing on the mean for $\delta^{\star}<3 \lambda_{1}{ }^{1 \star} / 2$ and $\delta^{\star}>3 \lambda_{1}{ }^{\star \star} / 2$ respectively; in the former $\boldsymbol{G}$ tends to align with the equilibrium orientation in the $\left(\boldsymbol{e}_{2}, \boldsymbol{e}_{3}\right)$ plane defined by $\zeta_{e q}$ and, pro- vided that $\zeta \simeq \zeta_{\text {eq }}$, the norm growth rate is unconditionally positive for $\delta^{\star}<0$. For $\delta^{\star}>0$ the norm growth rate is positive if $\left(1-r_{1}^{2}\right)^{1 / 2}>2 \delta^{\star} / 3-\lambda_{1}{ }^{\star \star}$ which again shows that, even if strain prevails, effective rotation tends to oppose the rise of the scalar gradient.

One can also consider the situation where vorticity aligns with $\boldsymbol{e}_{3}$, but in this case the equation for the azimuthal angle between the $\left(\boldsymbol{e}_{1}, \boldsymbol{e}_{2}\right)$ plane and $\boldsymbol{G}$ has no stable fixed point.

${ }^{1}$ A. Pumir, "A numerical study of the mixing of a passive scalar in three dimensions in the presence of a mean gradient," Phys. Fluids 6, 2118 (1994).

${ }^{2}$ Z. Warhaft, "Passive scalars in turbulent flows," Annu. Rev. Fluid Mech. 32, 203 (2000).

${ }^{3}$ P. Vedula, P. K. Yeung, and R. O. Fox, "Dynamics of scalar dissipation in isotropic turbulence: A numerical and modeling study," J. Fluid Mech. 433, 29 (2001).

${ }^{4}$ G. Brethouwer, J. C. R. Hunt, and F. T. M. Nieuwstadt, "Micro-structure and Lagrangian statistics of the scalar field with a mean gradient in isotropic turbulence," J. Fluid Mech. 474, 193 (2003).

${ }^{5}$ M. S. Chong, A. E. Perry, and B. J. Cantwell, "A general classification of three-dimensional flow fields," Phys. Fluid 2, 765 (1990).

${ }^{6} \mathrm{G}$. Erlebacher and S. Sarkar, "Statistical analysis of the rate of strain tensor in compressible homogeneous turbulence," Phys. Fluids A 5, 3240 (1993). ${ }^{7}$ K. Lee, S. S. Girimaji, and J. Kerimo, "Effect of compressibility on turbulent velocity gradients and small-scale structure," J. Turbul. 10, N9 (2009).

${ }^{8}$ D. H. Porter, P. R. Woodward, and A. Pouquet, "Inertial range structures in decaying compressible turbulent flows," Phys. Fluids 10, 237 (1998).

${ }^{9} \mathrm{H}$. Miura, "Analysis of vortex structures in compressible isotropic turbulence," Comput. Phys. Commun. 147, 552 (2002).

${ }^{10} \mathrm{~S}$. Pirozzoli and F. Grasso, "Direct numerical simulations of isotropic compressible turbulence: Influence of compressibility on dynamics and structures," Phys. Fluids 16, 4386 (2004).

${ }^{11}$ S. Suman and S. S. Girimaji, "Velocity gradient invariants and local flowfield topology in compressible turbulence," J. Turbul. 11, N2 (2010).

${ }^{12}$ B. L. Hua, J. C. McWilliams, and P. Klein, "Lagrangian acceleration in geostrophic turbulence,” J. Fluid Mech. 366, 87 (1998).

${ }^{13} \mathrm{P}$. J. Diamessis and K. K. Nomura, "Interaction of vorticity, rate of strain and scalar gradient in stratified, homogeneous sheared turbulence," Phys. Fluids 12, 1166 (2000).

${ }^{14}$ C. Pantano, S. Sarkar, and F. A. Williams, "Mixing of a conserved scalar in a turbulent reaction shear layer," J. Fluid Mech. 481, 291 (2003).

${ }^{15} \mathrm{~N}$. Swaminathan and R. W. Grout, "Interaction of turbulence and scalar fields in premixed flames," Phys. Fluids 18, 045102 (2006).

${ }^{16} \mathrm{~A}$. Mura, K. Tsuboi, and T. Hasegawa, "Modelling of the correlation between velocity and reactive scalar gradients in turbulent premixed flames based on DNS data," Combust. Theory Modell. 12, 671 (2008).

${ }^{17}$ N. Chakraborty and N. Swaminathan, "Influence of the Damköhler number on turbulence-scalar interaction in premixed flames. I. Physical insight," Phys. Fluids 19, 045103 (2007).

${ }^{18}$ G. Hartung, J. Hult, C. F. Kaminski, J. W. Rogerson, and N. Swaminathan, "Effect of heat release on turbulence and scalar-turbulence interaction in premixed combustion," Phys. Fluids 20, 035110 (2008).

${ }^{19}$ K. K. Nomura and S. E. Elghobashi, "The structure of inhomogeneous turbulence in variable density nonpremixed flames," Theor. Comput. Fluid Dyn. 5, 153 (1993).

${ }^{20}$ O. N. Boratav, S. E. Elghobashi, and R. Zhong, "On the alignment of the $\alpha$-strain and vorticity in turbulent nonpremixed flames," Phys. Fluids 8 , 2251 (1996)

${ }^{21}$ O. N. Boratav, S. E. Elghobashi, and R. Zhong, "On the alignment of strain, vorticity and scalar gradient in turbulent, buoyant, nonpremixed flames," Phys. Fluids 10, 2260 (1998).

${ }^{22}$ E. Dresselhaus and M. Tabor, "The kinematics of stretching and alignment of material elements in general flow fields," J. Fluid Mech. 236, 415 (1992).

${ }^{23}$ K. K. Nomura and G. K. Post, "The structure and dynamics of vorticity and rate of strain in incompressible homogeneous turbulence," J. Fluid Mech. 377, 65 (1998).

${ }^{24}$ G. Lapeyre, P. Klein, and B. L. Hua, "Does the tracer gradient vector align with the strain eigenvectors in 2D turbulence?," Phys. Fluids 11, 3729 (1999). 
${ }^{25}$ G. Lapeyre, "Topologie du mélange dans un fluide turbulent géophysique," Ph.D. thesis Université Paris VI, 2000.

${ }^{26} \mathrm{M}$. Gonzalez and P. Paranthoën, "On the role of unsteady forcing of tracer gradient in local stirring," Eur. J. Mech. B/Fluids 29, 143 (2010).

${ }^{27}$ G. R. Newman, B. E. Launder, and J. L. Lumley, "Modelling the behaviour of homogeneous scalar turbulence," J. Fluid Mech. 111, 217 (1981).

${ }^{28}$ A. Garcia and M. Gonzalez, "Analysis of passive scalar gradient alignment in a simplified three-dimensional case," Phys. Fluids 18, 058101 (2006).

${ }^{29}$ D. I. Pullin and T. S. Lundgren, "Axial motion and scalar transport in stretched spiral vortices," Phys. Fluids 13, 2553 (2001).

${ }^{30} \mathrm{~J}$. M. Burgers, "Application of a model system to illustrate some points of the statistical theory of free turbulence," Proc. R. Acad. Sci. Amsterdam 43, 2 (1940).

${ }^{31}$ T. S. Lundgren, "Strained spiral vortex model for turbulence fine structure," Phys. Fluids 25, 2193 (1982).

${ }^{32}$ W. T. Ashurst, A. R. Kerstein, R. M. Kerr, and C. H. Gibson, "Alignment of vorticity and scalar gradient with strain rate in simulated Navier-Stokes turbulence," Phys. Fluids 30, 2343 (1987).
${ }^{33} \mathrm{~K}$. Ohkitani, "Numerical study of comparison of vorticity and passive vectors in turbulence and inviscid flows," Phys. Rev. E 65, 046304 (2002).

${ }^{34}$ J. D. Gibbon and M. Heritage, "Angular dependence and growth of vorticity in the three-dimensional Euler equation," Phys. Fluids 9, 901 (1997).

${ }^{35}$ B. Galanti, J. D. Gibbon, and M. Heritage, "Vorticity alignment results for the 3D Euler and Navier-Stokes equations," Nonlinearity 10, 1675 (1997).

${ }^{36} \mathrm{~K}$. Ohkitani, "Eigenvalue problems in three-dimensional Euler flows," Phys. Fluids A 5, 2570 (1993).

${ }^{37}$ L. Chevillard, C. Meneveau, L. Biferale, and F. Toschi, "Modeling the pressure Hessian and viscous Laplacian in turbulence: comparisons with direct numerical simulation and implications on velocity gradient dynamics," Phys. Fluids 20, 101504 (2008).

${ }^{38} \mathrm{M}$. Gonzalez, "Kinematic properties of passive scalar gradient predicted by a stochastic Lagrangian model," Phys. Fluids 21, 055104 (2009).

${ }^{39}$ N. Peters, Turbulent Combustion Cambridge University Press, Cambridge, 2000.

${ }^{40} \mathrm{M}$. Gonzalez and P. Paranthoën "Effect of density step on stirring properties of a strain flow" Fluid Dyn. Res. 41, 035508 (2009). 